\title{
The Biomedical Functions and Related Mechanisms of Anthocyanin-Rich Extracts from Different Plant-Based Food
}

\author{
Jiayi Tang and Yixiao Shen* \\ College of Food Science, Shenyang Agricultural University, Shenyang, China \\ *Corresponding author: Yixiao Shen, College of Food Science, Shenyang Agricultural University, Shenyang, China. \\ To Cite This Article: Jiayi Tang, Yixiao Shen. The Biomedical Functions and Related Mechanisms of Anthocyanin-Rich Extracts from Different \\ Plant-Based Food. Am J Biomed Sci \& Res. 2021 - 12(5). AJBSR.MS.ID.001800. DOI: 10.34297/AJBSR.2021.12.001800.
}

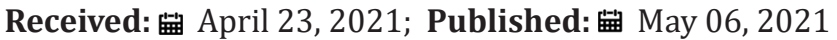

\begin{abstract}
Anthocyanins are a group of natural edible functional pigments, which not only provide the attractive color for plant, but also generate biomedical functions on various chronic diseases. Dietary interventions of anthocyanins are being investigated extensively which are associated with the prevention of chronic disorders and diseases. This review has summarized the latest research on the biomedical functions (including cholesterol control, asthma inflammation inhibition, retinal cells and liver fibrosis protection) of anthocyanin extracts from various food-based plants. The mechanisms of anthocyanin biomedical activity were attributed by the antioxidative, anti-inflammatory, and anti-proliferative properties, as well as the ability to regulate gene expression and metabolic pathways.
\end{abstract}

\section{Introduction}

Anthocyanins, a class of water-soluble pigments widely found in plants [1]. They have the typical structure of flavonoids and are derivatives of 2-phenylbenzocchidic cations with C6-C3-C6 as the basic backbone. Anthocyanins are glycoside derivatives of anthocyanidins. There are more than 300 different anthocyanins in nature. They were found in a variety of fruits and vegetables such as purple sweet potato, cranberry, cranberry, blueberry, grape, elderberry, black currant, purple carrot and red cabbage, ranging in color from red to blue [1]. The types of anthocyanins mainly include delchindin, cyanidin, petunidin and peonidin.

Anthocyanin has been already reported about its antioxidant ability and its effect on some chronic diseases. Epidemiological studies have found that long-term intake of plant foods rich in flavonoids can reduce the incidence of chronic cardiovascular diseases (CVD) and generate anti-mutation and anti-tumor activities [2]. For example, red wine anthocyanin extracts had strong inhibitory activity against the growth of colon cancer cells HCT-15 and gastric cancer cells AGS [3]. Also, anthocyanin-rich extracts have exhibited great performance on attenuating low density lipoproteins oxidation, lipid peroxidation, dyslipidemia and reduced levels of CVD molecular biomarkers as well as improved total plasma antioxidant capacity [4]. Since the exploration of anthocyanin bioactivity continuously progresses, more biomedical functions of anthocyanin have been discovered. Therefore, in this review, the latest research on the biomedical functions of anthocyanin extracts such as the protective effect on asthma, hyperlipidemia, retinal damage etc. and the related mechanisms were introduced and discussed. It will provide essential information for develop functional food or dietary supplements using anthocyanin extracts.

\section{Latest Biomedical Functions and Related Mechanisms of Anthocyanin Extracts}

\section{Anti-glycation function}

Advanced glycation end product (AGE) is a complex group of compounds that formed through Maillard reaction, a nonenzymatic reaction between free amino groups of protein and the carbonyl group of reducing sugar [5]. It is one of the important factors that induce diabetes and other chronic diseases. In the study 
of Wu et al (2009), mulberry anthocyanins were extracted and used in a BSA/glucose model system in order to investigate inhibitory effect of Mulberry anthocyanins on AGEs formation [6]. The results indicated that AGEs decreased significantly $(\mathrm{P}<0.05)$ with the increase of mulberry anthocyanin concentrations. The SDSPAGE electrophoretogram also showed that mulberry anthocyanin extract could inhibit the formation of protein crosslinking caused by glycosylation reaction. Meanwhile, the protein carbonyl and thiol groups content were significantly reduced with the treatment of mulberry anthocyanins [7]. It indicated that the mulberry anthocyanin extracts inhibited AGEs formation induced by hydroxyl radicals $(-\mathrm{OH})$ by protecting thiol and capturing reactive carbonyl intermediates.

\section{Cholesterol regulation}

The physiological activity of cholesterol metabolism of Lonicera caerule berry extracts (LCBE) has been evidenced in both in vitro and in vivo models. The effects of LCBE on cholesterol absorption and transport, as well as on the expression of genes controlling cholesterol synthesis, efflux, and absorption, were investigated in a Caco- 2 cell monolayer model. The results showed that 40 and $80 \mu \mathrm{g} / \mathrm{ml}$ of LCBE significantly reduced cholesterol absorption and transport, which further inhibited the cholesterol accumulation in a Caco-2 cell monolayer model [8]. Also, gene and protein expression of synthetic critical of cholesterol (SREBP2) and absorption (NPCIL1 and ACAT2) were significantly inhibited by LCBE $(\mathrm{p}<0.01)$. Furthermore, LCBE showed a potential function promoting cholesterol efflux through upregulation of mRNA and/ or protein levels of $\mathrm{ABCAl}$ andABCG5/G8 $(\mathrm{p}<0.01)$. It was therefore concluded that LCBE exhibited hypocholesterolemic performance via multiple mechanisms, including modulation of cholesterol biosynthesis, absorption, and efflux-related gene expression.

In the in vivo model, the hypolipidemic effect of LCBE was confirmed using high cholesterol diet (HCD)-induced hypercholesterolemia rats. The result demonstrated that LCBE treatment decreased body and organ weights significantly $(p<0.01)$ [8]. In addition, it normalized abnormal plasma lipoprotein levels and decreased liver cholesterol levels in hypercholesterolemic rats $(p<0.01)$. LCBE also improved histopathological features of the hepatic and heart aortas in rats fed an HCD. Compared to the HCD control group, LCBE treatment significantly decreased levels of total cholesterol, total glyceride, low-density lipoprotein cholesterol, increased high-density lipoprotein cholesterol. LCBE treatment promoted greater neutral and acidic sterol excretion $(p<0.01)$, and improved the antioxidant capacity of colon tissue, colon contents, and blood. Moreover, trimethylamine N-oxide (TMAO) levels also decreased in plasma ( $\mathrm{p}<0.01$ vs HCD group). On the molecular level, dietary LCBE treatment promoted the metabolism of cholesterol to bile acids and their efflux by activating PPARy-LXRa-ABCA1/ CYP7A1 pathways and downregulating the mRNA and protein expression of cholesterol biosynthesis gene SREEBP2 [9]. Moreover, NPC1L1, ACAT2, and MTP mRNA and protein expression were reduced, while ABCG5/8 expression increased $(\mathrm{p}<0.01)$ after LCBE treatment [8]. These results indicated that the therapeutic effect of LCBE is associated with efflux metabolism and reduced absorption of dietary cholesterol and biosynthesis through regulating the related genes.

\section{Asthma inflammation inhibition}

In recent years, inhibition of Th2 cell activation in the treatment of asthma has become a research concern. Anthocyanins are glycosides formed by the combination of anthocyanins and various sugars by sugar bonds. Generally, Th2 cells, mast cells, monocytes, lymphocytes, eosinophils and neutrophils are the main inflammatory cells, which release cytokines and play important roles in asthma [10]. These cytokines could also induce the release of histamine and leukotriene resulting in airway hyperresponsiveness. Specially, Th2 cytokines, such as L-4 and IL5 , regulate the body's immune response, inducing IgE production and chemotactic eosinophils [11]. Thus, the pathogenesis of asthma is related to the imbalance of Thl/Th2 cells. In the study of Arora et al (2016), the total number of cells in bronchitis alveolar lavage fluid and lymphocytes, eosinophils and neutrophils significantly decreased after anthocyanin extracts treatment in asthma mice [12]. In addition, asthma Th2 cytokines IL-4 and IL-5 levels in lung tissue of mice were significantly reduced. As a result, the anthocyanin extract inhibited the hyperresponsiveness of airways in mice and pulmonary inflammatory cell infiltration by inhibiting the Th2 cytokines IL-4 and IL-5.

\section{Retinal cells protection}

Bulberry anthocyanin extract protected retinal pigment epithelium (RPE) cells from damage induced by H2O2, as indicated by morphological observation [13]. Also, anthocyanins from blueberries are capable of reducing A2E-epoxidation by quenching singlet oxygen and protecting RPE cells from A2E cytotoxicity [14]. Apoptosis in the RPE cells treated by anthocyanin extracts decreased approximately three times compared with control group. Thus, the anthocyanin extracts could inhibit oxidative damage induced by H2O2 in RPE cells and change the expression of apoptotic factors through antioxidant activity.

\section{Liver fibrosis inhibition}

Effect of anthocyanin extracts on liver fibrosis in TGF$\boldsymbol{\beta}$-induced HSC-T6 cells: Anthocyanins can effectively inhibit aspartate aminotransferase (AST), alanine aminotransferase (ALT) and other liver function indicators [15]. Also, it reduces the mRNA 
level of inflammatory factors such as interleulin-1 (IL-1) and interleukin-6 (IL-6) and inhibit the expression of alpha smooth muscle motor protein ( $\alpha$-SMA) and other proteins [16].

Effect of anthocyanin extracts on liver fibrosis in mice: Anthocyanin extracts have a regulatory effect on the body weight of mice and has a significant inhibitory effect on the abnormal increase of liver coefficient in liver fibrosis mice [17]. It showed a significant protective effect on liver function indexes in the blood and liver tissue of liver fibrosis mice. Enhanced levels of antioxidant related enzymes (SOD, GSH-PX) also indicated that anthocyanin extracts can alleviate the symptoms of liver fibrosis in a dose-dependent manner [18]. Meanwhile, the expression of inflammatory factors (IL-1, IL-6, TNF-a COX-2) in liver tissue were inhibited. The inhibition of the expression of TGF- $\beta 1$ and P-Smad 2 protein in the TGF- $\beta$ / Smad signaling pathway were observed with the treatment of anthocyanin extract during liver fibrosis $[19,20]$. The above results suggested that inhibition of liver fibrosis by anthocyanin extracts may be achieved through the TGF- $\beta$ / Smad signaling pathway and reduce the accumulation of inflammation factors in liver tissue, thereby achieving the effect of delaying liver fibrosis.

\section{Conclusion}

It has been widely studied that anthocyanin extracts are associated with multiple bioactivities including scavenging free radical damage/oxidative stress, protecting cells, tissues and vital organs. This review has reported the latest biomedical functions of anthocyanin rich extracts such as antiglycation effect, cholesterol regulation, retinal cells protection and positive effect on asthma inflammation and liver fibrosis. It would provide essential information for consumers, healthcare providers, and the food and health industry to understand the relationship between anthocaynins and health. It is highly recommended that daily intake can mitigate the risk of diseases.

\section{Acknowledgement}

The review was supported by the Organization Department of Liaoning Province “Xingliao Talent Project” (XLYC1907107).

\section{Conflict of Interest}

The authors have no conflicts of interest to declare.

\section{References}

1. Mattioli R, Francioso A, Mosca L, Silva P (2020) Anthocyanins: A comprehensive review of their chemical properties and health effects on cardiovascular and neurodegenerative diseases. Molecules 25(17): 3809.

2. Pascual TS, Moreno DA, Garcia VC (2012) Flavonoid intake and cardiovascular disease mortality in a prospective cohort of US adults. Am J Clin Nutr 95(2): 454-464.
3. Dharmawansa KVS, Hoskin DW, Rupasinghe HPV (2020) Chemopreventive effect of dietary anthocyanins against gastrointestinal cancers: A review of recent advances and perspectives. Int J Mol Sci 21(18): 6555.

4. Reis JF, Monteiro VV, de Souza Gomes R, do Carmo MM, da Costa GV, et al. (2016) Action mechanism and cardiovascular effect of anthocyanins: A systematic review of animal and human studies. J Transl Med 14(1): 315

5. Chen JH, Lin X, Bu C, Zhang X (2018) Role of advanced glycation end products in mobility and considerations in possible dietary and nutritional intervention strategies. Nutr Metab (Lond) 15: 72.

6. Wu JW, Hsieh CL, Wang HY, Chen HY (2009) Inhibitory effects of guava (Psidium guajava L.) leaf extracts and its active compounds on the glycation process of protein. Food Chemistry. 113(1): 78-84.

7. Khalifa I, Xia D, Dutta K, Peng J, Jia Y, et al. (2020) Mulberry anthocyanins exert anti-AGEs effects by selectively trapping glyoxal and structuraldependently blocking the lysyl residues of B-lactoglobulins. Bioorg Chem 96: 103615.

8. Liu S, You L, Zhao Y, Chang X (2018) Wild Lonicera caerulea berry polyphenol extract reduces cholesterol accumulation and enhances antioxidant capacity in vitro and in vivo. Food Res Int 107:73-83.

9. Wang G, Han T, Wang S, Chen M, Sun Y, et al. (2018) Peroxisome proliferator-activated receptor- $\gamma$ prevents cholesterol gallstone formation in C57bl mice by regulating bile acid synthesis and enterohepatic circulation. Biomed Res Int 2018: 7475626.

10. Saglani S, Lloyd CM (2014) Eosinophils in the pathogenesis of paediatric severe asthma. Curr Opin Allergy Clin Immunol 14(2): 143-148.

11. Roufosse F (2018) Targeting the Interleukin-5 pathway for treatment of eosinophilic conditions other than asthma. Front Med 5: 49.

12. Arora P, Ansari SH, Najmi AK, Anjum V, Ahmad S (2016) Investigation of anti-asthmatic potential of dried fruits of Vitis vinifera L. in animal model of bronchial asthma. Allergy Asthma Clin Immunol 12: 42.

13. Wang Y, Guo X, Sun H, Qi W, Li A (2019) Bilberry anthocyanin-rich extract protects against retinal photooxidative damage via activation of HO-1 and inhibition of NF-кB. Food Agr Immunol 30(1): 829-840.

14. Bosch Morell F, Villagrasa V, Ortega T, Acero N, Muñoz Mingarro D, et al. (2020) Medicinal plants and natural products as neuroprotective agents in age-related macular degeneration. Neural Regen Res 15(12): 22072216.

15. Xia X, Wang X, Cheng Z, Qin W, Lei L, et al. (2019) The role of pyroptosis in cancer: pro-cancer or pro-"host"?. Cell Death Dis 10(9): 650.

16. Zhao J, Lin Y, Zhao Y, Wang Y, Ning C, et al. (2018) Polyphenol-rich blue honeysuckle extract alleviates silica particle-induced inflammatory responses and macrophage apoptosis via NRF2/HO-1 and MAPK signaling. J of Func Foods 46: 463-474.

17. Fan M, Choi YJ, Tang Y, Sung Mun Bae, Hyun Pil Yang, et al. (2019) Efficacy and mechanism of polymerized anthocyanin from grape-skin extract on high-fat-diet-induced nonalcoholic fatty liver disease. Nutrients. 11(11): 2586.

18. Park S, Zhang T, Qiu J, Wu X (2019) The combination of mulberry extracts and silk amino acids alleviated high fat diet-induced nonalcoholic hepatic steatosis by improving hepatic insulin signaling and nrmalizing gut microbiome dysbiosis in rats. Evid Based Complement Alternat Med 2019: 8063121.

19. Vugic L, Colson N, Nikbakht E, Gaizab A, Holland OJ, et al. (2020) Anthocyanin supplementation inhibits secretion of pro-inflammatory cytokines in overweight and obese individuals. J Funct Foods 64: 103596.

20. Park YJ, An HT, Park JS, Park O, Duh AJ, et al. (2020) Tyrosine kinase inhibitor neratinib attenuates liver fibrosis by targeting activated hepatic stellate cells. Sci Rep 10: 14756. 